\title{
On the Habitual and Occasional Senses of Mandarin ziji
}

\author{
Jianming Wu \\ Institute of Linguistics, Shanghai International Studies University, Shanghai, China
}

Email address:

wu.jianming2011@hotmail.com

\section{To cite this article:}

Jianming Wu. On the Habitual and Occasional Senses of Mandarin ziji. International Journal of Language and Linguistics. Vol. 8, No. 6, 2020, pp. 246-250. doi: 10.11648/j.ij11.20200806.12

Received: October 30, 2020; Accepted: November 11, 2020; Published: November 19, 2020

\begin{abstract}
In the literature, Mandarin ziji "self" has been fitted into various grammatical categories, such as reflexive pronoun, locally-bound anaphor, long-distance reflexive, adnominal/adverbial intensifier, etc. Different from previous grammatical analyses, the author argues that ziji, as a lexeme, has two types of senses, namely, the primary sense, i.e. "self" and the secondary sense, which includes speaker's reflection, speaker's identification, participant's reflection, unexpectedness, exclusion of others, focused identity, etc. These secondary senses not only help ziji to be linked to a referent in discourse but also distinguish it from the three-way personal pronouns in Mandarin. By resorting to a group of randomly-generated examples from the BCC corpus, the author finds out that speaker's reflection and speaker's identification are the most frequent secondary senses of ziji (each accounts for $81 \%$ or $61 \%$ of ziji), whereas participant's reflection (i.e. logophoricity) is the lest frequent one (i.e. $8 \%$ of $z i j i$ ). Thus, the author defines the former two secondary senses as 常义 habitual senses (i.e. senses that are most or very likely to be present when ziji is used in utterances) and the rest ones as 偶义 occasional senses (i.e. senses that are relatively less likely to be present when ziji is used in utterances). The significance of this demarcation lies in an understanding that there is always an asymmetrical distribution among the secondary senses of a lexeme, which, hopefully, predicts how it may be used or understood in the most relevant way.
\end{abstract}

Keywords: Primary Sense, Secondary Sense, Mandarin ziji, Reflexive

\section{Introduction}

In terms of Mandarin ziji, one fundamental question needs to be answered, that is, what does ziji mean when the speaker uses it? This question follows the insight originated from the Semiotic Triangle, which suggests that ziji "self", as a linguistic sign, is not directly linked to a referent, but via the intermediate stage of "senses" [1].

As we know, Mandarin ziji has no gender, number or person inflection. This is typical for words in isolating languages. Thus, unlike English himself or herself, ziji does not overtly express its referential values with regards to the speaker, hearer or the third party. In fact, it is an invariant form that needs to be substantiated with more specific referential values in context. In the process of production, the speaker conceptualizes the referent in his mind and verbalizes it with the lexeme of ziji; and in the process of comprehension, the hearer recognizes the senses of this lexeme and then associates it with the referent in discourse. It is these senses that we shall examine carefully in this paper.
The paper is structured as follows: Section 2 provides an understanding on the senses of a lexeme and classifies them into the primary sense and secondary sense; Section 3 divides the secondary senses of ziji into habitual senses and occasional senses; Section 4 discusses how the referent of $z i j i$ can be resolved with the aid of two habitual senses; finally, some concluding remarks are offered in Section 5. Most of the following examples, if not otherwise specified, come from the BCC corpus (http://bcc.blcu.edu.cn/).

\section{Classifying the Senses of a Lexeme}

Senses are viewed as the intermediate stage between a sign and its referent. Yet senses are notoriously difficult to grasp. A lexeme typically has one primary sense and multiple secondary senses. A primary sense is the sort of meaning that a dictionary would give for a word. It is usually the direct, basic or literal meaning of the word when it is referred to alone. For 
example, chair usually means a seat that has four legs and a back; run usually means going forward by springing steps; warm usually means a feeling of heat at a moderate temperature. These primary senses provide us with a conception as to how an entity, an action or a concept may be like, although the actual representation is not exactly the same among individuals or language communities.

Apart from the primary sense, a lexeme may have an array of secondary senses. A secondary sense is the meaning of a word when it is used with other words in context. The English functional word, but, is case in point. The primary sense of but, as a conjunction, is to connect two coordinate elements. However, in actual uses, but generally assumes several secondary senses that are extended from the primary one, as shown in (1):

(1) a. John is poor but he is honest. (contrast).

b. It never rains but pours (not concomitantly)

c. There is no hope but praying (only the other way)

d. Everyone but Tom has come in (except; saving)

e. But that is not possible! (surprised or annoyed)

Another case in point is the English word since. In the $15^{\text {th }}$ century, since is used to mean temporal sequence between two events. As a matter of fact, events described in temporal order often have a causal relation inferred. For instance, the sentence, John has been sad since Susan left him, may be read as "John has been sad from the time Suan left him" or "John has been sad because Susan left him" [2]. If the temporal reading of since was its primary sense in the $15^{\text {th }}$ century, an inferred causal reading should then be its secondary sense. Through frequency of usage in history, this previously inferred sense did become independent and was subsequently documented as part of the formal usage of a word. Today, the word since may also mean ago, later than, when, subsequently, etc.; it may even trigger a sense of angriness, as in since when did he ever listen to me? But this latter sense of angriness, which is also a secondary one, should be much less frequent than the temporal or causal one.

Therefore, there appear to be an unequal distribution of secondary senses that are associated with a word. The temporal or causal reading of since must be more frequent than the reading of angriness, and the contrast reading of but must be much more frequent than the reading of surprised or annoyed. This should reflect the native speakers' habit or preference in using these two words. The most frequent secondary sense of a word is likely to be the most relevant element in communication and consequently become the "positive cognitive effect", which is agreed upon by both the speaker and hearer [3]. Therefore, instead of treating every one of the secondary senses equally, we should differentiate the most frequent one from the less frequent ones. In this paper, the secondary senses that are the most frequent or very frequent in a sample are termed habitual senses (which should account for at least $60 \%$ of the cases) and the relatively less frequent ones (i.e. below 60\%) are termed occasional senses.

Then what could be the habitual senses of Mandarin ziji and what are the occasional ones? These questions will be addressed in the following section.

\section{The Habitual and Occasional Senses of Mandarin $z i j i$}

Regarding ziji, it is fair to say that the most direct or literal sense of it is "self" and nothing more. Yet the significance of this word lies in its secondary senses, i.e. meanings when it is used with other words in context. For instance, Huang [4][5] points out that that long-distance ziji conveys a message of "unexpectedness", as in (2):

(2) Xiaoming yiwei mama hui lai jie ziji.

Xiaoming think mum will come pick up self

"Xiaoming think that mum will pick up him (self)."

According to Huang, the choice of ziji, instead of ta "him", is licensed by the M-heuristic (i.e. "a marked message indicates a marked situation") [6]. In other words, if both ziji and $t a$ are used as person indexicals, ziji is the marked one in the above context. A marked form typically conveys some marked information, as Huang (ibid) also points out that "examined in a more careful way, this unexpectedness may turn out to be logophoricity, emphaticness/contrastiveness or something yet to be discovered". As far as this paper is concerned, although both ziji "self" and ta "him" refer to the same matrix subject, they differ a lot in their respective secondary senses. The $3^{\text {rd }}$ person pronoun, ta "he/she/him/her", is generally used to point to a referent from the speaker's standpoint with regards to the addressee or others (e.g. I \& you vs. they), while ziji does not directly refer to a speech-act participant but denotes, in the main, the internal thoughts or consciousness of the person it refers to. This is why a sense of unexpectedness may be triggered.

In terms of logophoricity, it is a source of reflection from the matrix subject in the sentence (i.e. Xiaoming is reflecting on himself, so his feeling of unexpectedness can be reported) [7]. In actual uses, ziji may denote two sources of reflection: one is from the main participant towards the participant himself, as in (2). The other is from the speaker towards the participant in the sentence, as in (3 a \&b).

(3) a. zuqiu ye hui zai buyuan de jianglai football too will at near MOD future zhanshi ziji juren de mianmao. reveal self giant MOD face

"Football will too reveal the face of a giant in the near future."

b. suanle, ni yi ge ren zhu zai waimian yao after all you one CL man live at outside need haohao zhaogu ziji.

well look after self

"After all, you live outside alone and need to take care of yourself well."

In (3a), although ziji attests an anaphoric relationship with the antecedent, zuqiu "football", it is usually not possible for "football" to be a human cognizer. However, the choice of ziji, instead of the $3^{\text {rd }}$ inanimate person $t a$ "it", actually personifies "football", which may be read as if it had an ego to be 
reflected upon by the speaker. A sense of emotional identification from the speaker may also arise, showing that the speaker is empathetic with the football game in his country. Similarly in (3b), the choice of ziji, instead of $n i$ "you", also convey a message that the speaker is reflecting on the participant and is being empathetic $[8,9]$.

What is more, ziji is also used to express a sense of "focused identity", which means the person being referred to is being emphatically pointed out by the speaker [10], as in (4).

(4) weiqilai de zhe pian zhaoze xianzhi de

Enclosing REL this CL swamp limit REL

shi cunmin ziji.

is villagers self

"Enclosing this piece of swamp harms the villagers themselves."

In (4), the intention to single out the villagers as some focused identities also brings about a sense of "comparison/contrast". In other words, the act of enclosing the wetland harms none other than the villagers themselves, while others who live outside this village would not be affected at all. This sense of "comparison/contrast" is associated with the nature of selfhood, i.e. the presence of self always indicates some significant others in the background [12, 13]. For example, in the following sentence, the situation that the subject $t \bar{a}$ "he" gave the hope of life to others while keeping the chance of death to himself is indeed a great contrast.

(5) ta ba sheng de xiwang liu gei bieren,

he PREP life MOD hope save for others

si de weixie liu gei ziji.

death MOD threat save for self

"He left the hope of life to others and the threat of death to himself."

This relational sense of ziji (i.e. contrast/comparison) also leads to the other secondary senses, such as "exclusion of others", "without help" "alone", etc., as in (6), where it says we have to rely on ourselves in the end, meaning excluding any intervening external forces.

(6) zhe ge shijie shui dou bu kekao

this CL world who all not dependable

zuizhong hai de quan kao ziji.

finally have to all depend self

"Anyone is not dependable in this world; (we) have to depend on ourselves in the end."

Finally, ziji also trigger a secondary sense of "generally-known" in (7). Typically, in the type of sentences, ziji refers to a group of non-specific people (e.g. we, you all or everyone) [14] and the sentence could be regarded as some folk wisdom or proverbial-like sayings.

(7) henduo shihou zhiyou ziji cai neng

many times only self only can

banzhu ziji.

help self

Lit. "Only self can help self most of the times."

To summarize, we have at least the following secondary senses of ziji, as shown in (8) below.

(8) a. speaker's reflection: ziji is understood to carry the speaker's reflection on a specific participant in the sentence (including the speaker himself).

b. speaker's identification: ziji is understood to carry the speaker's emotional identification towards a specific participant (including the speaker himself) in the sentence.

c. participant's reflection: ziji is understood as referring back to the matrix subject and carry its thoughts/consciousness from it.

d. focused identity: ziji triggers a sense that the entity being depicted has some distinguishing characteristics of its own or it is focused or emphasized.

e. comparison/contrast: ziji triggers a sense that the entity being depicted is contrastively different from the others.

f. unexpectedness: ziji triggers a sense that certain action, outcome or event has not been fully anticipated.

g. exclusion of others: ziji triggers a sense of certain action or state being depicted is without the intervention of others.

h. generally-known: ziji, which usually profiles a set of non-specific individuals, triggering a sense that the proposition related is commonly-known or generally applicable.

In order to examine how these secondary senses are distributed, we resort to a group of authentic examples collected from the BCC corpus (http://bcc.blcu.edu.cn/), which has a total of 15 billion words, including such genres as newspapers, novels, blog writings, science \& technology articles, etc. We collected 500 instances of ziji that were randomly generated by this corpus. Each of them is coded at least with one of the secondary senses mentioned above. Each sense should be agreed upon by the author and two other students during the coding process. All of the coders are native speakers of Chinese. The distribution of secondary senses is shown below.

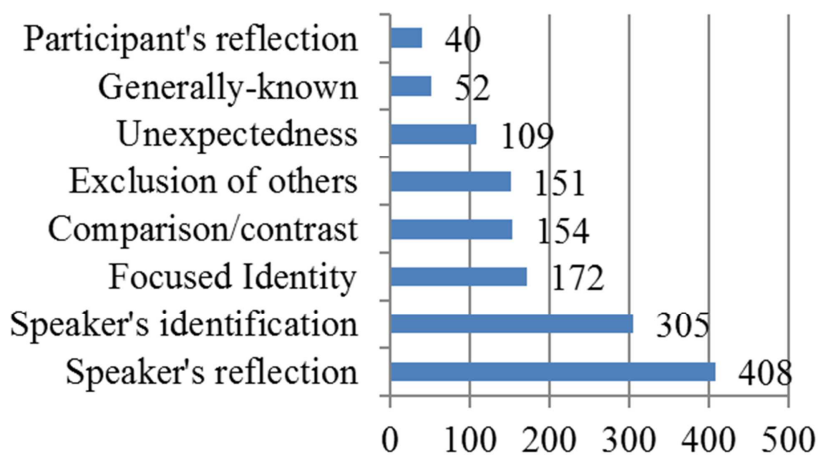

Figure 1. The distribution of secondary senses for Mandarin ziji.

According to Figure 1, two of the most frequent secondary senses of ziji are "speaker's reflection" (on himself or others) and "speaker's identification" (with himself or others). Thus, in a sample of 500 randomly selected ziji, the probability rates for ziji to be associated with "speaker's reflection" and "speaker's identification" are relatively higher than others, i.e. P 
(sp.re) $=0.81$ and $P($ sp.id $)=0.61$. In contrast, the probability rate for the sense of "participant's reflection" (i.e. logophoricity) is the lowest one, i.e. $\mathrm{P}$ (pa.re) $=0.08$.

Therefore, considering the relative probability rates of secondary senses in the sample, we propose that "speaker's reflection" and "speaker's identification" should be the habitual senses for Mandarin ziji, while the rest, which are detected in less than $60 \%$ of the cases, should be the occasional senses. Such a demarcation is useful because it reminds us that there is currently an asymmetrical distribution of secondary senses for a word, which, hopefully, help us predict how it is used in the most probable way.

In the next section, we will see how the habitual senses may help resolve the referent of ziji.

\section{The Relevance of Habitual Senses in Resolving the Referent of $z i j i$}

In the previous section, we mentioned that ziji is not directly linked to its referent but via the intermediate stage of senses. But how could this process of referencing be possible? To begin with, we may have a look at the structural domains where ziji may establish a linking relationship with its referent in the sample, as shown in Figure 2 below.

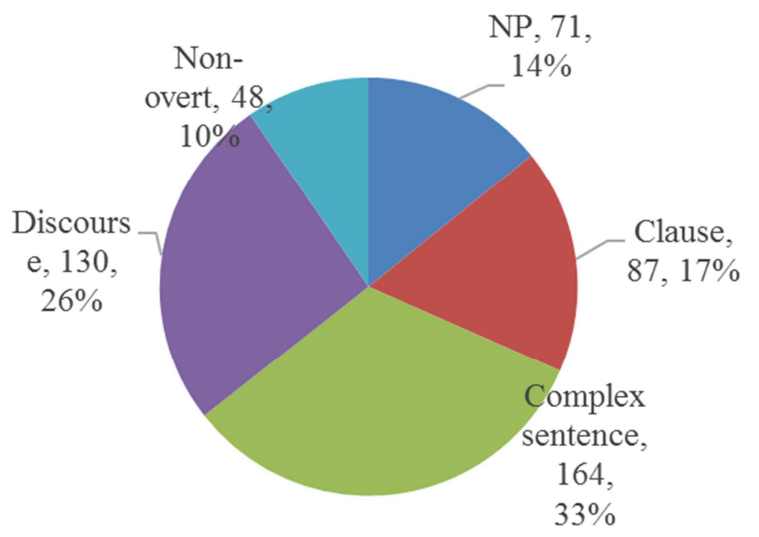

Figure 2. The structural domains where ziji is linked to a referent.

Figure 2 shows that there are four possible structural domains that ziji may be linked to a referent. They are the domain of an NP (i.e. ziji is used appositively to a noun or pronoun), a clause (i.e. ziji is the object of a transitive verb in a clause), a complex sentence (i.e. ziji appears in a subordinate clause and is viewed as a long-distance reflexive) and a discourse (i.e. the referent of ziji can be found in the neighboring sentences). We see that complex sentences are the more likely domain than others for ziji to assume coreference, which accounts for $33 \%$ of the cases, whereas clauses account only for $17 \%$ of the cases. This may suggest that locality [15], which is the essential structural property for English $x$-self, is not equally important or even unnecessary for Mandarin ziji. In fact, according to Figure 2, there is no structural domain that dominates the uses of ziji in the data, which means not a single structural/grammatical analysis is sufficient for ziji.
On the other hand, there are $26 \%$ of the instances of ziji that have a referent in the neighboring sentences (i.e. discourse) and $10 \%$ do not have an overt referent in any of the above structural domains. This also means the referent of ziji cannot be resolved by syntax only. But how could this be possible? We may look at the following sentences to see how this may happen.

(9) zhe ben shu shi yi ge nvhai gei ziji this CL book is a CL girl give self 20 sui shengri de liwu 20 years old birthday MOD gift

"A girl gave me a book as a gift for my 20th birthday."

"A girl gave herself a book as a gift for her 20th birthday."

In (9), the sentence may have two interpretations depending on the referent of ziji. One interpretation is that a girl gave herself a book on her $20^{\text {th }}$ birthday. This interpretation requires the speaker identifies himself with the girl and reports her thoughts/consciousness, i.e. logophoricity. The other interpretation is that a girl gave the speaker a book as a gift for the speaker's $20^{\text {th }}$ birthday. This interpretation requires the speaker to reflect on himself as a participant in the sentence. Although both interpretations are possible and grammatical in Chinese, Figure 1 suggests that the second interpretation, that is, the speaker's reflection on the participant including himself, is 7 times more likely than the first interpretation. Then most readers will think ziji refers to the speaker is most relevant and ziji has no antecedent in the sentence.

Let us look at another example quoted from the corpus, which has two interpretations as well.

(10) ta kuai yao bu neng huxi le,

She soon become not can breathe ASP, ta shi zhen de dasuan shasi ziji.

he is really plan to kill self

"She could not breathe. He really wanted to kill her."

In (10), the phrase ta dasuan shasi ziji "he plans to kill self" is a canonical reflexive context. In this context, it means the speaker's reflection on the participant, with or without a certain degree of emotional identification. However, quite contrary to our expectation, the context of the sentence tells us that the reason that "she cannot breathe" is because "he is trying to strangle her" ( $t a$ "he/she" is distinguished with Modern Chinese characters in gender but not in pronunciation). In other words, it is possible that the male subject is strangling the female subject, and she is worried that "he may kill herself". Then, the occasional sense of logophoricity is taking effect here. Since the probability rate of logophoricity is much lower than empathy, the second interpretation often produces some unexpectedness among Chinese readers as well (for the first interpretation is usually expected).

\section{Conclusion}

The above discussion has brought about some new analyses concerning Mandarin ziji. We proceed from the distinction between the primary and secondary senses and then 
classifying the latter into the habitual senses and occasional senses. Based on a sample collected from the BCC corpus, we regard "speaker's reflection" and "speaker's identification" as the habitual senses of ziji.

We believe that the referent of ziji cannot be resolved by syntax only. In fact, semantic/pragmatic factors, or more exactly, the habitual senses of ziji should play a big role. The significance of pinpointing the habitual senses of a word lies in the fact that there is always an asymmetrical distribution of secondary senses in the functional inventory of meanings for a word [16]. This helps us predict how a word may be used in the most relevant fashion. In the future, we attempt to look into what could be the constant senses of ziji, which is supposed to underlie every single use of ziji in Mandarin.

\section{References}

[1] C. K. Ogden and I. A. Richards, "The Meaning of Meaning: A Study of the Influence of Language upon Thought and of the Science of Symbolism," Vol. 29. K. Paul, Trench, Trubner \& Company, Limited, 1923.

[2] J. L. Bybee, "Diachronic linguistics," In The Oxford handbook of cognitive linguistics G. Dirk \& H. Cuyckens, Eds. New York: Oxford University Press. 2007.

[3] D. Wilson and D. Sperber, "Relevance theory," in The Handbook of pragmatics, L. Horn and G. Ward, Eds. Blackwell, Oxford, 2004.

[4] Y. Huang, The syntax and pragmatics of anaphora: A study with special reference to Chinese. Cambridge: Cambridge University Press, 1994.
[5] Y. Huang, Anaphora: Across-Linguistic Study. Oxford: Oxford University Press, New York. 2000.

[6] S. Levinson, Presumptive Meanings. Cambridge, MA: The MIT Press. 2000.

[7] Sells, P. (1987). Aspects of logophoricity. Linguistic inquiry, 18 (3): 445-479.

[8] S. Kuno, Functional Syntax: Anaphora, Discourse, and Empathy. Chicago: The University of Chicago Press. 1987.

[9] Oshima, D. Y. (2004). Zibun revisited: empathy, logophoricity, and binding. Proceedings of the 20th NWLC, 23: 175-190.

[10] König, E. and Gast, V. (2006). Focused assertion of identity: A typology of intensifiers. Linguistic Typology, 10 (2), 223-276.

[11] König, E., \& Siemund, P. (2000). The development of complex reflexives and intensifiers in English. Diachronica, 17 (1): 39-84.

[12] Andersen, S. M., Chen, S., \& Miranda, R. (2002). Significant others and the self. Self \& Identity, 1 (2): 159-168.

[13] Brewer, M. B. and Gardner, W. (1996). Who is this" We"? Levels of collective identity and self representations. Journal of personality and social psychology, 71 (1): 83.

[14] Siewierska, A. (2008). Introduction: Impersonalization from a subject-centred vs. agent-centred perspective. Transactions of the Philological Society, 106 (2): 115-137.

[15] N. A. Chomsky, Lectures on government and binding. Dordrecht: Foris, 1981.

[16] Wu, J. (2019). The inventory structure of Person in the Chinese dialect of Puxian. Language and Linguistics, 20 (4): 631-655. 